\title{
EXPERIMENTAL IDENTIFICATION OF GHM AND ADF PARAMETERS FOR VISCOELASTIC DAMPING MODELING
}

\author{
C. M. A. Vasques ${ }^{1}$, R. A. S. Moreira ${ }^{2}$ and J. Dias Rodrigues ${ }^{1}$ \\ ${ }^{1}$ Faculdade de Engenharia da Universidade do Porto \\ Departamento de Engenharia Mecânica e Gestão Industrial \\ Rua Dr. Roberto Frias s/n, 4200-465 Porto, Portugal \\ E-mail: \{cvasques,jdr\}@fe.up.pt \\ ${ }^{2}$ Universidade de Aveiro \\ Departamento de Engenharia Mecânica \\ Campus Santiago, 3810-193 Aveiro, Portugal \\ E-mail: rmoreira@mec.ua.pt
}

Keywords: Viscoelastic, measurement, complex modulus, GHM, ADF, finite element, damping, 3M ISD112.

\begin{abstract}
The implementation of the Golla-Hughes-McTavish (GHM) and Anelastic Displacement Fields $(A D F)$ models in a general finite element (FE) model with viscoelastic damping is presented and discussed in this paper. Additionally, a direct frequency analysis (DFA) is also described and employed. A methodology to identify the complex shear modulus of the viscoelastic materials is described. The identified complex shear modulus of the viscoelastic material 3M ISD112 is curve-fitted in order to obtain the modeling parameters of the GHM and ADF models. A sandwich plate with a viscoelastic core and elastic skins is analyzed. Measured and predicted frequency response functions (FRFs) are compared with the purpose of assessing the performance of the presented damping models. The analysis allows to asses the validity of the methodology to determine the frequency dependent complex modulus, the GHM and ADF parameters identification and the outcomes and drawbacks of the DFA, GHM and ADF viscoelastic damping modeling strategies.
\end{abstract}

\section{INTRODUCTION}

Nowadays, in certain technological areas (e.g., automotive, aeronautics, aerospace, acoustics) there is a growing need of lighter and stiffer structural components. However, the weight reduction, maintaining the rigidity unaffected, might lead to a significant reduction of the inherent damping capacity of those flexible structures. Viscoelastic materials can be used as an effective means of controlling the dynamics of structures, reducing and controlling the structural vibrations and noise radiation from structures. They can be used in surface mounted or embedded damping treatments, utilizing passive viscoelastic materials alone, the so-called passive treatments, or in an unified way with active materials such as piezoelectrics, the so-called 
hybrid treatments. The use of these materials in damping treatments provides high damping capability over wide temperature and frequency ranges.

Applications with passive treatments alone, have been widely used since the end of the 1950s, as can be found in reference textbooks (e.g., [1-4]). However, hybrid treatments are a class of treatments usually more expensive and difficult to implement, but with the advantage of leading to a higher damping performance. Therefore, a considerable research effort has been undertaken towards their effective use in real life applications. A review of hybrid treatments is addressed in [5-8] and some analytical and experimental studies concerning beams, plates and shells can be found, for example, in [9-18].

In the last decade the technology associated with a more refined design and adequate implementation of viscoelastic-based damping treatments has achieved a relative maturity and is starting to be frequently applied in practice by the scientific community and its industrial partners. In order to adequately design these viscoelastic damping treatments the viscoelastic material properties need to be accurately measured and considered for the underlying mathematical models. The temperature and frequency dependent material properties of the viscoelastic materials complicate the mathematical model and make the solution of the problem more difficult to obtain. Usually, isothermal conditions are assumed and only the frequency dependent constitutive behavior is taken into account. The simplest way of modeling these materials is achieved by a complex modulus approach (CMA) where the material properties are defined for each discrete frequency value $[19,20]$. The CMA is also associated with the so-called modal strain energy (MSE) method [21] where the loss factors of each individual mode are determined from the ratios between the dissipated modal strain energy of the viscoelastic counterpart and the storage modal strain energy of the global structural system. The MSE method is known to lead to poor viscoelastic damping estimation of highly damped structural systems. Furthermore, the CMA is a frequency domain method that is limited to steady state vibrations and single-frequency harmonic excitations. Thus, to account for the frequency dependent material properties, iterative versions of the MSE have been used successfully for moderate damping values [22]. Time domain models, relying on internal variables (see [23]), such as the Golla-Hughes-McTavish (GHM) [24, 25] and anelastic displacement fields (ADF) [26, 27], or others [28, 29], utilizing additional dissipation variables, have been successfully utilized and yield good damping estimates. Alternatively, the use of fractional calculus (FC) [30, 31] models, based upon the use of fractional derivatives, has the drawback of generating a "non-standard" finite element (FE) formulation, with a more complex characteristic solution procedure, but yielding also good damping estimates. Other relevant contribution for the viscoelastic damping modeling is given by Adhikari's work [32] and his references therein. Regarding the temperature effects, studies taking into account the temperature dependence of the properties and the self-heating of viscoelastic materials have been performed by Lesieutre and his co-workers [33, 34] which extended the ADF model for these cases leading, however, to nonlinear differential equations. The effects of the operating temperature on hybrid damping treatments performance and viscoelastic damping efficiency were analyzed, for example, in [35-39].

The extensive use of passive or hybrid treatments using viscoelastic materials to reduce vibration and sound radiation from structures has motivated the integration of the aforementioned viscoelastic damping models into commercial or home-made FE codes, which is a very common tool for studying complex structures used by the major part of the structural designers. Time domain models represent better alternatives to CMA-based models allowing the reduction of the computational burden and the study of the transient response in a more straightforward manner, even for highly damped structural systems. Among the time domain models, internal 
variables models are more interesting from the computational point of view and easiness of implementation into FE codes. Thus, the GHM and ADF models are alternative methods, used to model the damping behavior of viscoelastic materials in $\mathrm{FE}$ analysis, which yield a standard FE formulation (however with the addition of some "non-physical" dissipative variables). In order to use them, one needs the GHM and ADF characteristic parameters which allow characterizing the complex (frequency dependent) constitutive behavior of the viscoelastic material being used. To do that, experimental procedures to measure the isotropic constitutive behavior (usually the shear modulus) and numerical identification procedures of the measured data need to be developed.

In the open literature works concerning the experimental measurement of the viscoelastic constitutive behavior by different approaches (test configurations) can be found, for example, in $[1,4,40]$ and the references therein, where viscoelastic material master curves, tables and empirical and fractional derivative analytical expressions of the complex modulus and shift factor are presented for several types of viscoelastic materials. As referred by Jones [4], that information is provided from various sources and is fairly accurate, in general, but it is by no means the best data which can be obtained. It is, however, the best data available in the public domain, and better data ("real" data) may be obtained only by additional testing or purchase of data from a data base (e.g, [41, 42]), often at considerable cost.

This paper addresses the experimental identification of the GHM and ADF parameters to be used for modeling the viscoelastic damping behavior. The viscoelastic material considered is this work is the 3M ISD112 [43], which was chosen because of its commercial availability, although the methodology described below can be applied to any viscoelastic material. As reported by Trindade and Benjeddou [7] these parameters, in general, are adjusted by a curvefitting of the viscoelastic material master curves, in order to minimize the difference between the measured and estimated data. Lesieutre and Bianchini [26] presented the curve-fitting of the $3 \mathrm{M}$ ISD112 material data, at a temperature of $27^{\circ} \mathrm{C}$, in the frequency range $[8-8000] \mathrm{Hz}$. They concluded that five ADF (with two parameters per ADF) represent exactly the behavior of the material shear modulus and the loss factor. Friswell, Inman, and Lam [44] presented the same analysis for the GHM model, with three or four parameters per model. They used the $3 \mathrm{M}$ ISD112 material at $20^{\circ} \mathrm{C}$ in the frequency-range $[10-4800] \mathrm{Hz}$ and DYAD601 material at $24^{\circ} \mathrm{C}$ in the frequency range $[2-4800] \mathrm{Hz}$. Their results indicated that the model with four parameters represented the material data better than that with three parameters. In general, GHM and ADF models fit well the master curves of materials whose properties have strong frequency dependence. Nevertheless, the number of parameters needed is somehow related with the degree of frequency dependence of the material properties and usually increases with that dependence. That is why Enelund and Lesieutre [45] proposed a combination of the ADF model with the fractional derivatives model, where the relaxation equation for the anelastic strain is taken as an in time differential equation of fractional-order, combining the best features of the ADF model and the FC. It was shown that this fractional-order ADF model can predict the instantaneous transient response of the material over a wide frequency range using a single anelastic strain field (only one ADF serie) governed by a fractional-order equation with the drawback of yielding a "non-standard" FE formulation requiring a more complex characteristic solution procedure typical of FC.

In this work the implementation of the GHM and ADF models in a FE model is first presented and discussed. Next, the experimental test rig and methodology used to identify the complex shear modulus of the viscoelastic material 3M ISD112 is described. An analytical empirical expression for the complex modulus and the shift factor obtained by curve fitting master 
curves of 3M ISD112 is given in [40]. However, the accuracy of these expressions is not clear and with time manufacturers change the production process, with implications in the properties of the viscoelastic materials, making old data not so appropriate. Thus, that justifies the new experimental methodology to estimate new data presented in this work. Then, the curve-fitted viscoelastic material data is compared with the measured one and the parameters of the GHM and ADF models are presented and used to represent the frequency dependent viscoelastic stiffness and to model the viscoelastic damping behavior of the structure. The identified parameters are then utilized in a FE model analysis and validated by comparison of frequency response functions (FRFs) measured in a sandwich plate with a viscoelastic core and elastic skins.

The main contributions and novelties of this work are related with the fact that both measured and predicted results are utilized to validate the methodologies to include viscoelastic damping in FE models. Additionally, a new experimental procedure to determine the viscoelastic material properties is presented and discussed, and the characteristic material parameters of the GHM and ADF models of the 3M ISD112 are obtained by curve-fitting the measured shear storage modulus and loss factor of "actual" 3M ISD112 material. Furthermore, the experimental data is usually taken by a "manual" procedure from the plot of the nomogram given by the manufacturer. Moreover, Trindade et al. [22] compared the GHM, ADF and interactive MSE models, however without comparing the predicted results with any measured (real) or predicted results obtained by a direct frequency analysis (DFA) approach, which is the most processing time consuming technique, however the most precise. Therefore, the methodologies are assessed and compared in a more rigorous way.

\section{VISCOELASTIC DAMPING MODELING}

\subsection{Linear Viscoelasticity}

Viscoelastic materials are rubber-like polymers possessing stiffness and damping characteristics which vary strongly with temperature and frequency. This polymers are materials composed of long intertwined and cross-linked molecular chains, each containing thousands or even millions of atoms. The internal molecular interactions that occur during deformation in general, and vibration in particular, give rise to macroscopic properties such as stiffness and energy dissipation during cyclic deformation, which in turn characterizes the mechanism of viscoelastic damping.

It is well known that the viscoelastic constitutive behavior relies on the hypotheses that the current value of the stress tensor depends upon the complete past history of the components of the strain tensor. Thus, considering an isotropic viscoelastic material under isothermal conditions, the theory of linear viscoelasticity [46] states that the constitutive relationship (in shear or extension) for a one-dimensional stress-strain system can be written as

$$
\sigma(t)=G(t) \varepsilon(0)+\int_{0}^{t} G(t-\tau) \frac{\partial \varepsilon(\tau)}{\partial \tau} d \tau
$$

where the strain history $\varepsilon(t)$ is restricted to be zero for $t<0, \sigma(t)$ is the resultant stress (shear or extensional) and $G(t)$ is a constitutive time varying relaxation function. This gives rise to a hysteretic or complex modulus description for the vibration of a structure with viscoelastic material components. Thus, considering initial conditions equal to zero, i.e., $\varepsilon(0)=0$, the Laplace transform of Equation (1) yields

$$
\tilde{\sigma}(s)=s \tilde{G}(s) \tilde{\varepsilon}(s),
$$


Table 1: GHM and ADF relaxation functions.

\begin{tabular}{cc}
\hline Function & Author (year) \\
\hline$h(s)=\sum_{i=1}^{n} \hat{\alpha}_{i} \frac{s^{2}+2 \hat{\zeta}_{i} \hat{\omega}_{i} s}{s^{2}+2 \hat{\zeta}_{i} \hat{\omega}_{i} s+\hat{\omega}_{i}^{2}}$ & Golla, McTavish and Hughes (1985, 1993)[24, 25] \\
$h(s)=\sum_{i=1}^{n} \frac{s \Delta_{i}}{s+\Omega_{i}}$ & Lesieutre (1992) [48]
\end{tabular}

where $s \tilde{G}(s)$ is a material modulus function which in turn can be represented by the equality $s \tilde{G}(s)=G_{0}[1+h(s)]$ and substituted into (2) yielding

$$
\tilde{\sigma}(s)=G_{0}[1+h(s)] \tilde{\varepsilon}(s),
$$

where $G_{0}$ is the relaxed (or static) shear modulus obtained after the material relaxation, i.e., $G_{0}=\lim _{t \rightarrow \infty} G(t)$. Thus, since $G_{0}$ is constant, the term $G_{0} \tilde{\varepsilon}(s)$ represents the recoverable (elastic) counterpart of the material stress while $G_{0} h(s) \tilde{\varepsilon}(s)$ represents the non-recoverable energy dissipation effects (relaxation). For that reason, $h(s)$ is usually termed as dissipation or relaxation function.

Many authors have proposed different mathematical representations of the relaxation behavior $h(s)$ in the last decades, which in turn leads to different mathematical models of the viscoelastic damping behavior and different solution methods of the governing equations. The different relaxation functions are summarized in [7, 24, 32, 47] as a result of a compilation from several works, where the different models and solution methods of the resultant mathematical models are thoroughly discussed. Some of the most well known frequently referred in the open literature are the internal variables GHM and ADF models, which will be considered in this work and described in the following sections, and the correspondent relaxation functions are presented in Table 1.

\subsection{Complex Modulus Approach}

There are practical situations in which structures with viscoelastic materials may be subjected to steady-state oscillatory conditions. Under these conditions, assuming $s$ as pure imaginary variable, $s=j \omega$, the previously derived stress-strain relationship $s \tilde{G}(s)$ in Equation (2), referring to the shear constitutive term, might be expressed as a function of the frequency $\omega$ in the complex form

$$
G(j \omega)=G^{\prime}(\omega)+j G^{\prime \prime}(\omega),
$$

where $G^{\prime}(\omega)$ is the shear storage modulus, which accounts for the recoverable energy, and $G^{\prime \prime}(\omega)$ is the shear loss modulus, which represents the energy dissipation effects $[1,4,46]$. The loss factor of the viscoelastic materials is defined as

$$
\eta(\omega)=\frac{G^{\prime \prime}(\omega)}{G^{\prime}(\omega)},
$$

which alternatively allows to write Equation (4) as

$$
G(j \omega)=G^{\prime}(\omega)[1+j \eta(\omega)]
$$


For a linear, homogeneous and isotropic viscoelastic material, equivalent representations of the previous equations hold for the extensional modulus $E(j \omega)$ and the relationship

$$
G(j \omega)=\frac{E(j \omega)}{2[1+\nu(j \omega)]},
$$

where $\nu(j \omega)$ is the Poisson's ratio, establishes a relationship between the two. However, for simplicity, a real frequency independent Poisson's ratio $\nu(j \omega)=\nu$ is assumed, which turns the loss factors of the shear and extensional complex moduli equal, i.e., $\eta_{E}(\omega)=\eta_{G}(\omega)=\eta(\omega)$.

\subsection{Finite Element Implementation}

\subsubsection{Direct Frequency Analysis Response Model}

Considering the frequency dependent physical properties of the viscoelastic materials presented in the previous sections, the general time dependent FE equations of motion of a viscoelastically damped general structural elastic system can be written as

$$
\mathbf{M} \ddot{\mathbf{u}}(t)+\mathbf{D} \dot{\mathbf{u}}(t)+\left[\mathbf{K}^{E}+\mathbf{K}^{V}(j \omega)\right] \mathbf{u}(t)=\mathbf{F}(t),
$$

where $\mathbf{M}$ and $\mathbf{D}$ are the global mass and viscous proportional damping matrices, $\mathbf{K}^{E}$ and $\mathbf{K}^{V}(j \omega)$ are the elastic and viscoelastic stiffness matrices, and $\mathbf{u}(t)$ and $\mathbf{F}(t)$ are the displacements degrees of freedom (DoFs) and applied loads vectors. It's worthy to mention that two types of damping models are considered in the previous equation: a frequency dependent hysteretic (viscoelastic) damping type, represented by $\mathbf{K}^{V}(j \omega)$, whose terms are complex and frequency dependent, representative of the viscoelastic relaxation behavior; and a viscous proportional damping type, which is described by $\mathbf{D}$, representing other general sources of damping (e.g., the material damping of the elastic part of the structural system, air-based damping, energy dissipation in the supports, etc.), which are assumed to be proportional to the velocity.

Using Equation (8) as it is, the frequency dependent matrix definition implies its use and analysis only in the frequency domain, based in the CMA, where the material properties of the stiffness matrix of the viscoelastic layers are defined for each discrete frequency value [20,49]. Thus, the CMA is a frequency domain method where the frequency response model (FRFs) can be generated in a straightforward manner from the results of many discrete frequency calculations of the equations of motion, in which the complex stiffness matrix of the viscoelastic layers is re-calculated at each frequency value of the desired discrete frequency range.

Considering simple harmonic mechanical excitation,

$$
\mathbf{F}(t)=\overline{\mathbf{F}} e^{j \omega t},
$$

where $\overline{\mathbf{F}}$ is the vector of applied loads amplitudes, the steady state mechanical harmonic response of the system can be written as

$$
\mathbf{u}(t)=\overline{\mathbf{U}} e^{j \omega t},
$$

where $\overline{\mathbf{U}}$ is the vector response of displacement amplitudes. Substituting (9) and (10) into (8) yields

$$
\left[\mathbf{K}(j \omega)+j \omega \mathbf{D}-\omega^{2} \mathbf{M}\right] \overline{\mathbf{U}}=\overline{\mathbf{F}},
$$

where $\mathbf{K}(j \omega)=\mathbf{K}^{E}+\mathbf{K}^{V}(j \omega)$, from which the complex solution vector $\overline{\mathbf{U}}$ can be obtained. 
The FRF (frequency response function) for a load applied in the $i$ th DoF and a displacement DoF output $o$, can be obtained by solving (11) for different values of frequency,

$$
\left[\mathbf{K}\left(j \omega_{l}\right)+j \omega_{l} \mathbf{D}-\omega_{l}^{2} \mathbf{M}\right] \overline{\mathbf{U}}\left(j \omega_{l}\right)=\overline{\mathbf{F}}_{i},
$$

where $\overline{\mathbf{F}}_{i}$ denotes a force vector with a non-zero component in the $i$ th DoF and all other elements equal to zero, and $\overline{\mathbf{U}}\left(j \omega_{l}\right)$ is the resulting complex response vector (displacements) solution at frequency $\omega_{l}$. Thus, the receptance FRF at frequency $\omega_{l}, H_{o i}\left(j \omega_{l}\right)$, is given by

$$
H_{o i}\left(j \omega_{l}\right)=\frac{\bar{U}_{o}\left(j \omega_{l}\right)}{\bar{F}_{i}}
$$

where $\bar{F}_{i}$ is the amplitude of the load input and $\bar{U}_{o}\left(j \omega_{l}\right)$ is the displacement amplitude extracted from the oth DoF of the vector $\overline{\mathbf{U}}\left(j \omega_{l}\right)$.

Finally, the FRF can be generated from the results of many discrete frequency calculations of Equation (12), in which the complex stiffness matrix of the viscoelastic layers is re-calculated at each frequency value of the discrete frequency range $\omega=\omega_{0}, \ldots, \omega_{l}, \ldots, \omega_{f}$, as depicted in Figure 1.

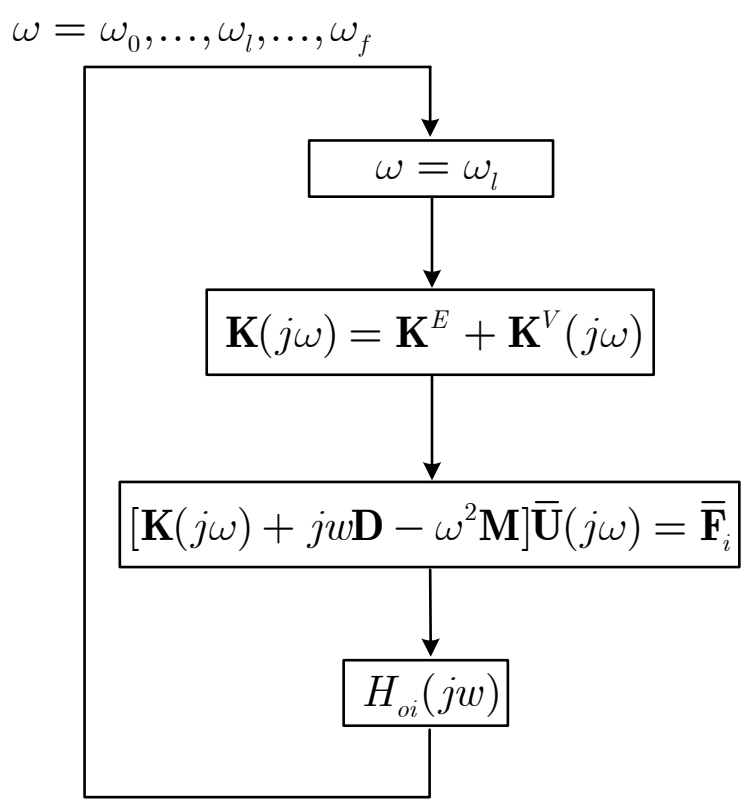

Figure 1: Direct frequency analysis (DFA) response model generation diagram.

\subsubsection{GHM Damping Model}

When general transient responses are required, time domain models are more suitable and versatile and represent better alternatives to the CMA since they allow the reduction of the computational burden due to the recalculation of the stiffness matrix for each discrete frequency value. One alternative is the GHM (Golla-Hughes-McTavish) model [24, 25] which assumes the material modulus function $s \tilde{G}(s)$ represented in terms of a series of mini-oscillator terms (Table 1),

$$
s \tilde{G}(s)=G_{0}[1+h(s)]=G_{0}\left(1+\sum_{i=1}^{n} \hat{\alpha}_{i} \frac{s^{2}+2 \hat{\zeta}_{i} \hat{\omega}_{i} s}{s^{2}+2 \hat{\zeta}_{i} \hat{\omega}_{i} s+\hat{\omega}_{i}^{2}}\right),
$$


where $G_{0}$ is the relaxed modulus and each $i$ th mini-oscillator term is a second order rational function involving three positive constant parameters $\hat{\alpha}_{i}, \hat{\omega}_{i}$ and $\hat{\zeta}_{i}$. These parameters govern the shape of the modulus function over the complex plane (or over the frequency domain assuming only the imaginary part of the complex plane), and depending on the nature of the viscoelastic material modulus function and the range of $s$ (or frequency) over which it is to be modeled, the number of mini-oscillator terms is defined according to the accuracy of the representation required.

Considering the FE equations of motion of a viscoelastically damped general structural elastic system in Equation (8), with the shear modulus (note that both shear and extensional stiffness terms were considered in the viscoelastic stiffness matrix through the extensional and shear modulus relationship in (7) assuming a frequency independent Poisson's coefficient) of the viscoelastic counterpart factored out of the viscoelastic stiffness matrix, yielding $\overline{\mathbf{K}}^{V}$, and assuming that the structural system possess only one type of viscoelastic material, Equation (8) following the hereditary stress-strain law in (1) yields

$$
\mathbf{M} \ddot{\mathbf{u}}(t)+\mathbf{D} \dot{\mathbf{u}}(t)+\mathbf{K}^{E} \mathbf{u}(t)+G(t) \overline{\mathbf{K}}^{V} \mathbf{u}(0)+\int_{0}^{t} G(t-\tau) \overline{\mathbf{K}}^{V} \frac{\partial \mathbf{u}(\tau)}{\partial \tau} d \tau=\mathbf{F}(t) .
$$

In a similar way to what has been considered in Section 2.1, transforming Equation (19) to the Laplace domain yields

$$
\left(s^{2} \mathbf{M}+s \mathbf{D}+\mathbf{K}^{E}\right) \tilde{\mathbf{u}}(s)+s \tilde{G}(s) \overline{\mathbf{K}}^{V} \tilde{\mathbf{u}}(s)=\tilde{\mathbf{F}}(s) .
$$

Substituting the material modulus function representation in Equation (14) into (16) yields

$$
\left(s^{2} \mathbf{M}+s \mathbf{D}+\mathbf{K}^{E}\right) \tilde{\mathbf{u}}(s)+G_{0} \overline{\mathbf{K}}^{V}\left(1+\sum_{i=1}^{n} \hat{\alpha}_{i} \frac{s^{2}+2 \hat{\zeta}_{i} \hat{\omega}_{i} s}{s^{2}+2 \hat{\zeta}_{i} \hat{\omega}_{i} s+\hat{\omega}_{i}^{2}}\right) \tilde{\mathbf{u}}(s)=\tilde{\mathbf{F}}(s) .
$$

Then, introducing a set of $n$ series of dissipation (or internal) variables $\tilde{\mathbf{u}}_{i}^{D}(s)(i=1, \ldots, n)$, for each series, one can assume the relationship

$$
\tilde{\mathbf{u}}(s)-\tilde{\mathbf{u}}_{i}^{D}(s)=\frac{s^{2}+2 \hat{\zeta}_{i} \hat{\omega}_{i} s}{s^{2}+2 \hat{\zeta}_{i} \hat{\omega}_{i} s+\hat{\omega}_{i}^{2}} \tilde{\mathbf{u}}(s) .
$$

Substituting Equation (18) into (17) yields

$$
\left(s^{2} \mathbf{M}+s \mathbf{D}+\mathbf{K}^{E}+\mathbf{K}^{V 0}\right) \tilde{\mathbf{u}}(s)+\mathbf{K}^{V 0} \sum_{i=1}^{n} \hat{\alpha}_{i}\left(\tilde{\mathbf{u}}(s)-\tilde{\mathbf{u}}_{i}^{D}(s)\right)=\tilde{\mathbf{F}}(s),
$$

where $\mathbf{K}^{V 0}=G_{0} \overline{\mathbf{K}}^{V}$ is the relaxed (static) stiffness matrix of the viscoelastic components. Next, in order to describe the dissipative behavior of the internal DoFs, after some algebra Equation (18) can be written as

$$
\tilde{\mathbf{u}}_{i}^{D}(s)=\frac{\hat{\omega}_{i}^{2}}{s^{2}+2 \hat{\zeta}_{i} \hat{\omega}_{i} s+\hat{\omega}_{i}^{2}} \tilde{\mathbf{u}}(s) .
$$

Therefore, considering Equations (19) and (20), after some algebra we get the following augmented coupled system,

$$
\begin{gathered}
\left(s^{2} \mathbf{M}+s \mathbf{D}+\mathbf{K}^{E}+\mathbf{K}^{V \infty}\right) \tilde{\mathbf{u}}(s)-\mathbf{K}^{V 0} \sum_{i=1}^{n} \hat{\alpha}_{i} \tilde{\mathbf{u}}_{i}^{D}(s)=\tilde{\mathbf{F}}(s), \\
\left(s^{2} \frac{1}{\hat{\omega}_{i}^{2}}+s \frac{2 \hat{\zeta}_{i}}{\hat{\omega}_{i}}+1\right) \tilde{\mathbf{u}}_{i}^{D}(s)-\tilde{\mathbf{u}}(s)=\mathbf{0}
\end{gathered}
$$


where

$$
\mathbf{K}^{V \infty}=\left(1+\sum_{i=1}^{n} \hat{\alpha}_{i}\right) \mathbf{K}^{V 0} .
$$

In order to obtain the time-dependent behavior of the augmented system, multiplying Equation (21b) by $\hat{\alpha}_{i} \mathbf{K}^{V 0}$ and since all matrices are independent of $s$, a linear time domain model is easily recovered by the inverse Laplace transform of Equations (21), yielding

$$
\begin{gathered}
\mathbf{M} \ddot{\mathbf{u}}(t)+\mathbf{D} \dot{\mathbf{u}}(t)+\left(\mathbf{K}^{E}+\mathbf{K}^{V \infty}\right) \overline{\mathbf{u}}(t)-\mathbf{K}^{V 0} \sum_{i=1}^{n} \hat{\alpha}_{i} \overline{\mathbf{u}}_{i}^{D}(t)=\mathbf{F}(t), \\
\frac{\hat{\alpha}_{i}}{\hat{\omega}_{i}^{2}} \mathbf{K}^{V 0} \ddot{\mathbf{u}}_{i}^{D}(t)+\frac{2 \hat{\alpha}_{i} \hat{\zeta}_{i}}{\hat{\omega}_{i}} \mathbf{K}^{V 0} \dot{\mathbf{u}}_{i}^{D}(t)+\hat{\alpha}_{i} \mathbf{K}^{V 0} \overline{\mathbf{u}}_{i}^{D}(t)-\hat{\alpha}_{i} \mathbf{K}^{V 0} \overline{\mathbf{u}}(t)=\mathbf{0} .
\end{gathered}
$$

The augmented coupled system in Equations (22) might also be expressed in compact matrix form as

$$
\overline{\mathbf{M}} \ddot{\overline{\mathbf{q}}}(t)+\overline{\mathbf{D}} \dot{\overline{\mathbf{q}}}(t)+\overline{\mathbf{K}} \overline{\mathbf{q}}(t)=\overline{\mathbf{F}}(t),
$$

where

$$
\begin{gathered}
\overline{\mathbf{M}}=\left[\begin{array}{cc}
\mathbf{M} & \mathbf{0} \\
\mathbf{0} & \mathbf{M}_{D D}
\end{array}\right], \overline{\mathbf{D}}=\left[\begin{array}{cc}
\mathbf{D} & \mathbf{0} \\
\mathbf{0} & \mathbf{D}_{D D}
\end{array}\right], \overline{\mathbf{K}}=\left[\begin{array}{cc}
\mathbf{K}_{E E} & \mathbf{K}_{E D} \\
\mathbf{K}_{D E} & \mathbf{K}_{D D}
\end{array}\right], \\
\overline{\mathbf{q}}(t)=\operatorname{col}\left[\overline{\mathbf{u}}(t), \overline{\mathbf{u}}_{1}^{D}(t), \ldots, \overline{\mathbf{u}}_{n}^{D}(t)\right], \overline{\mathbf{F}}(t)=\operatorname{col}[\mathbf{F}(t), \mathbf{0}, \ldots, \mathbf{0}],
\end{gathered}
$$

and

$$
\begin{gathered}
\mathbf{M}_{D D}=\operatorname{diag}\left(\frac{\hat{\alpha}_{1}}{\hat{\omega}_{1}^{2}} \mathbf{K}^{V 0}, \ldots, \frac{\hat{\alpha}_{n}}{\hat{\omega}_{n}^{2}} \mathbf{K}^{V 0}\right), \mathbf{D}_{D D}=\operatorname{diag}\left(\frac{2 \hat{\alpha}_{1} \hat{\zeta}_{1}}{\hat{\omega}_{1}} \mathbf{K}^{V 0}, \ldots, \frac{2 \hat{\alpha}_{n} \hat{\zeta}_{n}}{\hat{\omega}_{n}} \mathbf{K}^{V 0}\right), \\
\mathbf{K}_{D D}=\operatorname{diag}\left(\hat{\alpha}_{1} \mathbf{K}^{V 0}, \ldots, \hat{\alpha}_{1} \mathbf{K}^{V 0}\right), \mathbf{K}_{E D}=\left[-\hat{\alpha}_{1} \mathbf{K}^{V 0}, \ldots,-\hat{\alpha}_{n} \mathbf{K}^{V 0}\right], \\
\mathbf{K}_{E E}=\mathbf{K}^{E}+\mathbf{K}^{V \infty}, \mathbf{K}_{D E}=\mathbf{K}_{E D}^{\mathrm{T}} .
\end{gathered}
$$

\subsubsection{ADF Damping Model}

A time domain model based on a variation, or Laplace transformed, formulation of the ADF (Anelastic Displacement Fields) model, originally proposed by Lesieutre and his co-workers $[26,27]$, is proposed in this work. It takes a definition of a complex modulus in the frequency (or Laplace) domain and utilizes the so-called internal, dissipation or anelastic (after Lesieutre) variables to simplify the equations, although with the drawback of increasing the size of the problem. Afterward, through an inverse Laplace transform one obtains an amenable and computationally tractable augmented system of linear ordinary differential equations that can be solved by standard (linear) numerical procedures. With this procedure the FE model implementation of the ADF model is more straightforward when compared with the Lesieutre's original direct time domain formulation based on the methods of irreversible thermodynamics and a decomposition of the total displacement field in an elastic and anelastic counterpart.

Therefore, the process of deriving the augmented coupled elastic-anelastic (using the original designation of Lesieutre) is similar to what has been presented for the GHM method, however utilizing a different definition of the material modulus function, and only some key steps will be presented here. Thus, as reported by Lesieutre, the frequency dependent viscoelastic shear 
modulus described by the ADF model, represented by a series of functions in the Laplace domain, has the form $[26,48]$ ( $c f$. Table 1)

$$
s \tilde{G}(s)=G_{0}[1+h(s)]=G_{0}\left(1+\sum_{i=1}^{n} \frac{\Delta_{i} s}{s+\Omega_{i}}\right),
$$

where again $G_{0}=\lim _{t \rightarrow \infty} G(t)$ is the relaxed (or static, low-frequency) shear modulus, $\Omega_{i}$ is the inverse of the characteristic relaxation time at constant strain and $\Delta_{i}$ the correspondent relaxation resistance. To take into consideration the relaxation behavior, the entire ADF model itself may be comprised of several individual fields where $n$ series of ADF are used to describe the material behavior. Given measured values of the shear modulus in the form of frequency dependent complex modulus $G(j \omega)$, the relaxed shear modulus $G_{0}$ and the series of material parameters $\Delta_{i}$ and $\Omega_{i}$ can be determined using curve fitting or optimization techniques. The number of series of ADF parameters determines the accuracy of the matching of the measured data over the frequency range of interest.

Substituting Equation (24) into (16) yields

$$
\left(s^{2} \mathbf{M}+s \mathbf{D}+\mathbf{K}^{E}\right) \tilde{\mathbf{u}}(s)+G_{0} \overline{\mathbf{K}}^{V}\left(1+\sum_{i=1}^{n} \frac{\Delta_{i} s}{s+\Omega_{i}}\right) \tilde{\mathbf{u}}(s)=\tilde{\mathbf{F}}(s) .
$$

Then, in a similar way, introducing a set of $n$ series of anelastic (or internal, dissipation) $\tilde{\mathbf{u}}_{i}^{A}(s)$ $(i=1, \ldots, n)$ variables, for each series, one can assume the relationship

$$
\tilde{\mathbf{u}}(s)-\tilde{\mathbf{u}}_{i}^{A}(s)=\frac{s}{s+\Omega_{i}} \tilde{\mathbf{u}}(s) .
$$

Substituting Equation (26) into (25), considering the dissipative behavior of the anelastic DoFs given by from Equation (26) as

$$
\tilde{\mathbf{u}}_{i}^{A}(s)=\frac{\Omega_{i}}{s+\Omega_{i}} \tilde{\mathbf{u}}(s),
$$

after some algebra one gets the following augmented elastic-anelastic coupled system,

$$
\begin{gathered}
\left(s^{2} \mathbf{M}+s \mathbf{D}+\mathbf{K}^{E}+\mathbf{K}^{V \infty}\right) \tilde{\mathbf{u}}(s)-\mathbf{K}^{V 0} \sum_{i=1}^{n} \Delta_{i} \tilde{\mathbf{u}}_{i}^{A}(s)=\tilde{\mathbf{F}}(s), \\
\left(\frac{s}{\Omega_{i}}+1\right) \tilde{\mathbf{u}}_{i}^{A}(s)-\tilde{\mathbf{u}}(s)=\mathbf{0},
\end{gathered}
$$

where this time

$$
\mathbf{K}^{V \infty}=\left(1+\sum_{i=1}^{n} \Delta_{i}\right) \mathbf{K}^{V 0} .
$$

The time-dependent behavior of the augmented system, multiplying Equation (28b) by $\Delta_{i} \mathbf{K}^{V 0}$, is recovered by the inverse Laplace transform of Equations (28), yielding

$$
\begin{gathered}
\mathbf{M} \ddot{\mathbf{u}}(t)+\mathbf{D} \dot{\mathbf{u}}(t)+\left(\mathbf{K}^{E}+\mathbf{K}^{V \infty}\right) \overline{\mathbf{u}}(t)-\mathbf{K}^{V 0} \sum_{i=1}^{n} \Delta_{i} \overline{\mathbf{u}}_{i}^{A}(t)=\mathbf{F}(t), \\
\frac{\Delta_{i}}{\Omega_{i}} \mathbf{K}^{V 0} \dot{\mathbf{u}}_{i}^{A}(t)+\Delta_{i} \mathbf{K}^{V 0} \overline{\mathbf{u}}_{i}^{A}(t)-\Delta_{i} \mathbf{K}^{V 0} \overline{\mathbf{u}}(t)=\mathbf{0} .
\end{gathered}
$$


The augmented coupled system in Equations (29) might be expressed in compact matrix form as in Equation (23) where for the ADF model one gets

$$
\begin{aligned}
& \overline{\mathbf{M}}=\left[\begin{array}{cc}
\mathbf{M} & \mathbf{0} \\
\mathbf{0} & \mathbf{0}
\end{array}\right], \quad \overline{\mathbf{D}}=\left[\begin{array}{cc}
\mathbf{D} & \mathbf{0} \\
\mathbf{0} & \mathbf{D}_{A A}
\end{array}\right], \overline{\mathbf{K}}=\left[\begin{array}{cc}
\mathbf{K}_{E E} & \mathbf{K}_{E A} \\
\mathbf{K}_{A E} & \mathbf{K}_{A A}
\end{array}\right], \\
& \overline{\mathbf{q}}(t)=\operatorname{col}\left[\overline{\mathbf{u}}(t), \overline{\mathbf{u}}_{1}^{A}(t), \ldots, \overline{\mathbf{u}}_{n}^{A}(t)\right], \overline{\mathbf{F}}(t)=\operatorname{col}[\mathbf{F}(t), \mathbf{0}, \ldots, \mathbf{0}],
\end{aligned}
$$

where

$$
\begin{aligned}
& \mathbf{D}_{A A}=\operatorname{diag}\left(\frac{\Delta_{1}}{\Omega_{1}} \mathbf{K}^{V 0}, \ldots, \frac{\Delta_{n}}{\Omega_{n}} \mathbf{K}^{V 0}\right), \mathbf{K}_{A A}=\operatorname{diag}\left(\Delta_{1} \mathbf{K}^{V 0}, \ldots, \Delta_{n} \mathbf{K}^{V 0}\right), \\
& \mathbf{K}_{E E}=\mathbf{K}^{E}+\mathbf{K}^{V \infty}, \mathbf{K}_{E A}=\left[-\Delta_{1} \mathbf{K}^{V 0}, \ldots,-\Delta_{n} \mathbf{K}^{V 0}\right], \mathbf{K}_{A E}=\mathbf{K}_{E A}^{\mathrm{T}} .
\end{aligned}
$$

\subsubsection{State Space Design and Model Reduction}

As can be seen in Equations (22) and (29), the main disadvantage of GHM and ADF models is that, associated with a FE discretization and in order to account for the frequency dependence of the viscoelastic material, they lead to large systems, since they add auxiliary DoFs, which for each GHM or ADF series must be equal to the number of elastic DoFs. However, model reduction techniques might be utilized in order to reduce the size of the system. As suggested in [22] the matrices corresponding to the internal DoFs might be reduced and diagonalized through a projection in a suitable reduced modal base, to reduce the computational cost. The advantages of this alternative (transformed) representation are that in the case where only some part of the structure has surface mounted or embedded viscoelastic layers, only some FEs have viscoelastic components and $\mathbf{K}^{V 0}$ can have several rows and columns of zeros, which in turn leads to some nill eigenvalues. Thus, through an adequate coordinate transformation based on the eigenvalues and eigenvectors of $\mathbf{K}^{V 0}$ and elimination of the nil (spurious) eigenvalues the size of the problem can be substantially smaller. Furthermore truncated complex state space modal models might also be used in order to reduce the system size even further. Moreover, one may notice from $\bar{M}$ that when compared with the GHM model the anelastic DoFs of the ADF model have no inertia and therefore the global mass matrix $\bar{M}$ is singular and is not positivedefinite. However, the singularity of the mass matrix can be overcome if, instead of solving the second-order system (23) of the ADF, a state-space representation with an adequate design of the state variables is considered. The number of flexible modes is kept the same and the dissipative modes, which correspond to the internal relaxations of the viscoelastic material, are overdamped with low observability. The reader is referred to [22, 47, 50, 51] for further details about state space design and model reduction techniques.

\section{EXPERIMENTAL IDENTIFICATION OF VISCOELASTIC MATERIALS}

\subsection{Underlying Analytical Model}

The proposed experimental methodology for the dynamic characterization of the complex modulus of viscoelastic materials is based on the direct identification of the complex equivalent stiffness $K(j \omega)$ of a discrete single degree of freedom (SDoF) system [52]. The complex stiffness is physically materialized by a thin viscoelastic layer which is subjected to cyclic shear deformation imposed by a dynamic exciter. The dynamic response of the considered SDoF system is then used to evaluate the shear storage modulus and loss factor variation with frequency and temperature. 
The receptance FRF of the SDoF system, assuming stationary harmonic motion, is given by

$$
H(j \omega)=\frac{\bar{X}}{\bar{F}}=\frac{1}{K(j \omega)-\omega^{2} M}
$$

where $\bar{F}$ and $\bar{X}$ are the amplitudes of the dynamic force and displacement response and $M$ is the mass. The complex value stiffness $K(j \omega)$ of the viscoelastic sample can be directly determined through the inverse of the receptance, the dynamic stiffness function $Z(j \omega)=H^{-1}(j \omega)$, as

$$
K(j \omega)=\omega^{2} M+Z(j \omega) .
$$

The viscoelastic material storage modulus $G^{\prime}(\omega)$ and loss factor $\eta(\omega)$ functions can then be evaluated from

$$
\begin{gathered}
G^{\prime}(\omega)=\frac{h}{A_{S}}\left(\omega^{2} M-\operatorname{Re}[Z(j \omega)]\right), \\
\eta(\omega)=\frac{\operatorname{Im}[Z(j \omega)]}{\omega^{2} M+\operatorname{Re}[Z(j \omega)]},
\end{gathered}
$$

where $h$ is the thickness of the viscoelastic sample and $A_{S}$ its shear area.

\subsection{Experimental Apparatus}

The test rig representing the dynamic SDoF system used to identify the complex shear modulus of viscoelastic materials is illustrated in Figure 2. The underlying analytical model of the complex SDoF system previously described comprises a moving mass $M$, represented by the upper bar (2), and a complex stiffness $K(j \omega)$, represented by the thin viscoelastic layer sample (4) introduced between two rigid blocks, as shown in Figure 3. The upper bar is guided by two thin lamina springs (3), which provide the restraint of the spurious DoFs, allowing the viscoelastic specimen to deform mainly in shear due to the relative motion between the moving bar (2) and the fixed bar (1).

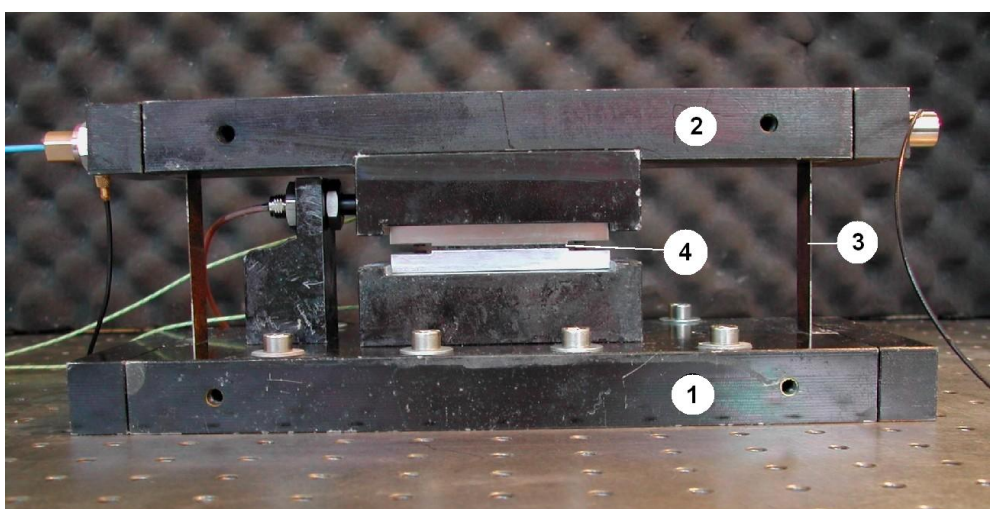

Figure 2: Test rig used to identify the complex shear modulus.

The dynamic excitation of the moving bar is provided by an electrodynamic shaker (Ling Dynamic Systems - model 201), using a thin stinger to minimize the rotation and lateral excitation, driven by a random signal generated by the signal generator of a dynamic signal analyzer (Brüel \& Kjær - model 2035) and amplified at a power amplifier (Ling Dynamic Systems - PA25E). The applied excitation force is measured using a piezoelectric force transducer (Brüel \& Kjær 


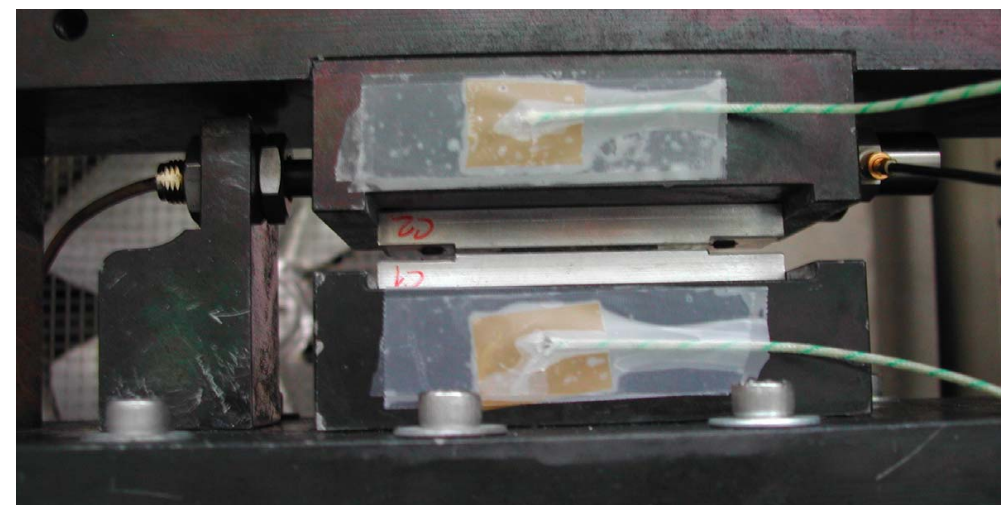

Figure 3: Detailed view of the viscoelastic specimen.

- model 8200). The acceleration response of the moving mass is measured with a piezoelectric accelerometer (Brüel \& Kjær - model 4371) and the displacement response with a proximity probe (Philips PR6423). The signal conditioning and the frequency response functions are determined with the aforementioned dynamic signal analyzer.

The test rig and the electrodynamic shaker are rigidly assembled on an inertial block (Figure 4), which is supported by rubber pads in order to minimize the influence of the rigid body modes of the assembly. Furthermore, the experimental apparatus was introduced in a thermal chamber, providing near isothermal conditions between 0 and $35^{\circ} \mathrm{C}$, with the temperature range limited by the shaker admissible range of operating temperature. The analysis at various temperatures allows the use of the temperature-frequency equivalence principle [4] extending the frequency range of the characterization and allowing data correlation. The temperature of the viscoelastic material is evaluated using two thermocouple probes located near the specimen, as depicted in Figure 3.

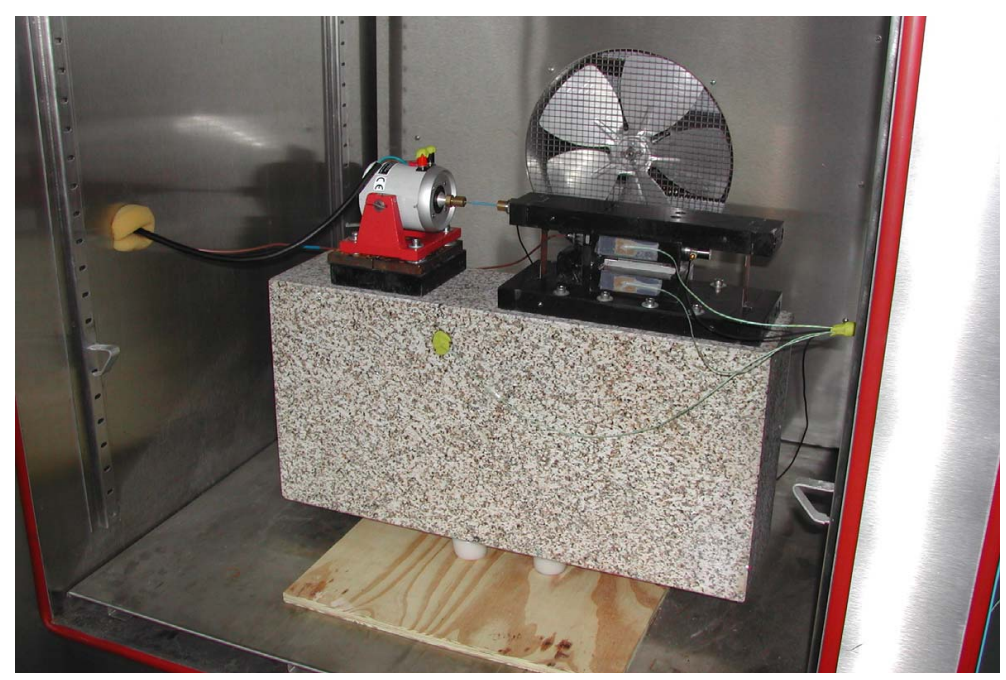

Figure 4: Experimental apparatus inside the thermal chamber.

\subsection{Numerical Analysis of Measured Complex Modulus Data}

The receptance and accelerance FRFs of the SDoF dynamic system described in the previous sections are measured using the transducer response signals (proximity probe, accelerometer 
and force transducer) for different temperatures. Since two different FRFs were measured using two different transducer responses (displacement and velocity), the corresponding FRFs can be correlated in order to identify high frequency noise generated by the thin springs, the stinger and shaker trunnion (highly evidenced by the receptance results), and the rigid body modes effects at low frequencies (evidenced in the accelerance results). Therefore, using both transducers, the useful frequency range is enlarged.

Viscoelastic damping is a property exhibited by a wide variety of polymeric materials, ranging from natural or synthetic rubber to industrial plastics, or even cork. The class of polymer materials is extremely wide and many polymeric compounds, displaying somewhat different complex modulus properties, available from commercial manufacturers and other sources (see $[1,4]$ for manufacturers) can be found. Some commonly available commercially viscoelastic damping polymers are the ISD110, 112 and 113 series, from 3M ${ }^{\mathrm{TM}}$, DYAD601, 606 and 609 series, from Soundcoat ${ }^{\circledR}$, to name just a few. Here attention is given to the 3M ISD112, which is the viscoelastic material identified and utilized in the damping treatment of the analyzed case study. However, as obvious, the present experimental procedure might be used to any other type of viscoelastic material.

The receptance and accelerance FRFs of a $0.57 \mathrm{~mm} \times 480 \mathrm{~mm}^{2} 3 \mathrm{M}$ ISD112 viscoelastic material specimen, measured in a bandwidth of $[0-400] \mathrm{Hz}$ at nine different temperatures between 2.7 and $33.5^{\circ} \mathrm{C}$ are presented in Figure 5. The obtained FRFs, whether using directly the receptance or the accelerance, are the inputs for the complex modulus description calculated using Equations (32).
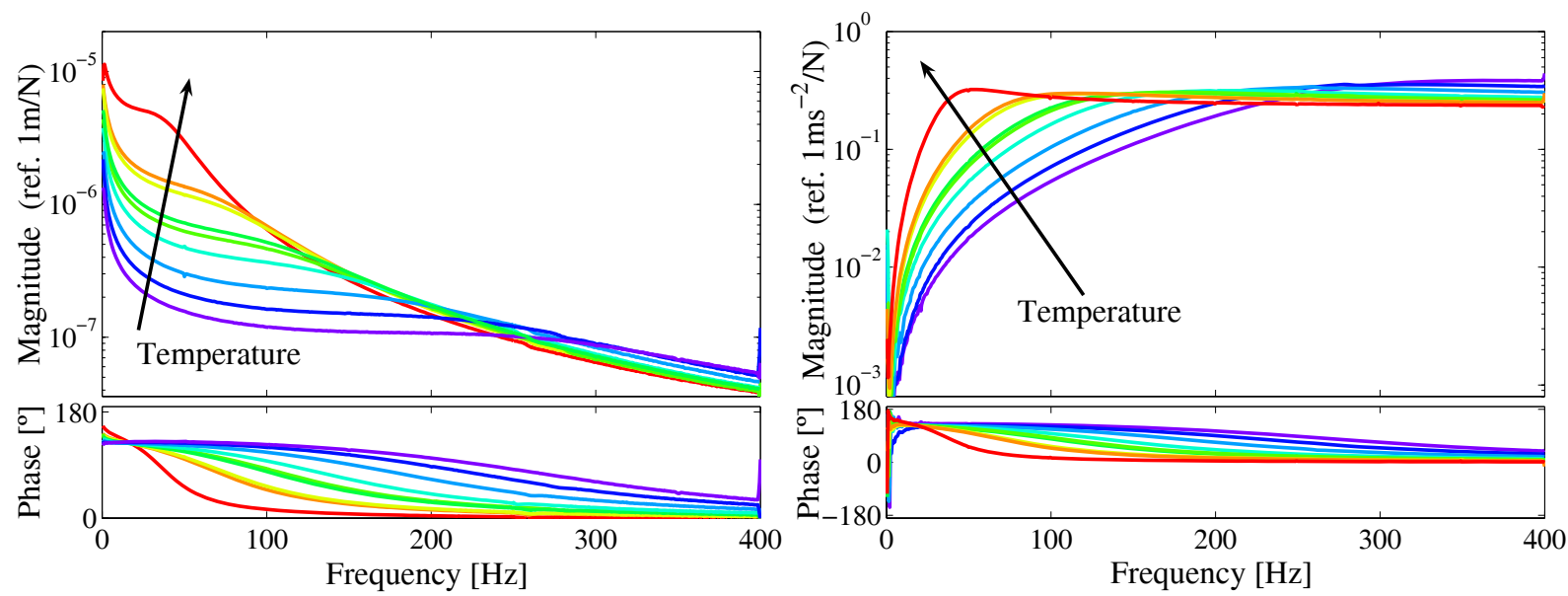

Figure 5: Measured receptance and accelerance FRFs at 2.7, 5.8, 7.1, 11.2, 14.4, 14.5, 18.8, 21.5 and 33.5 ${ }^{\circ} \mathrm{C}$.

In order to identify and filter possible random errors due to the high frequency noise, rigid body modes or other error sources, the identified complex modulus functions are plotted point by point in a Wicket Plot which is a useful graphical representation to highlight the errors (see [4] for further details). In this graphical representation the storage modulus calculated from the data at different temperatures are plotted against the corresponding loss modulus values. Figure 6 (a) represents the Wicket plot of the identified complex modulus data of the 3M ISD112 specimen. It stands out the master curve of the complex modulus, being possible to identify erroneous data sets, and the data lying outside the main sequence is eliminated. After filtering the data, removing all scattered points, a reliable data set of complex modulus is obtained, as illustrated in Figure 6 (b), which can be used to characterize the viscoelastic material under analysis. 

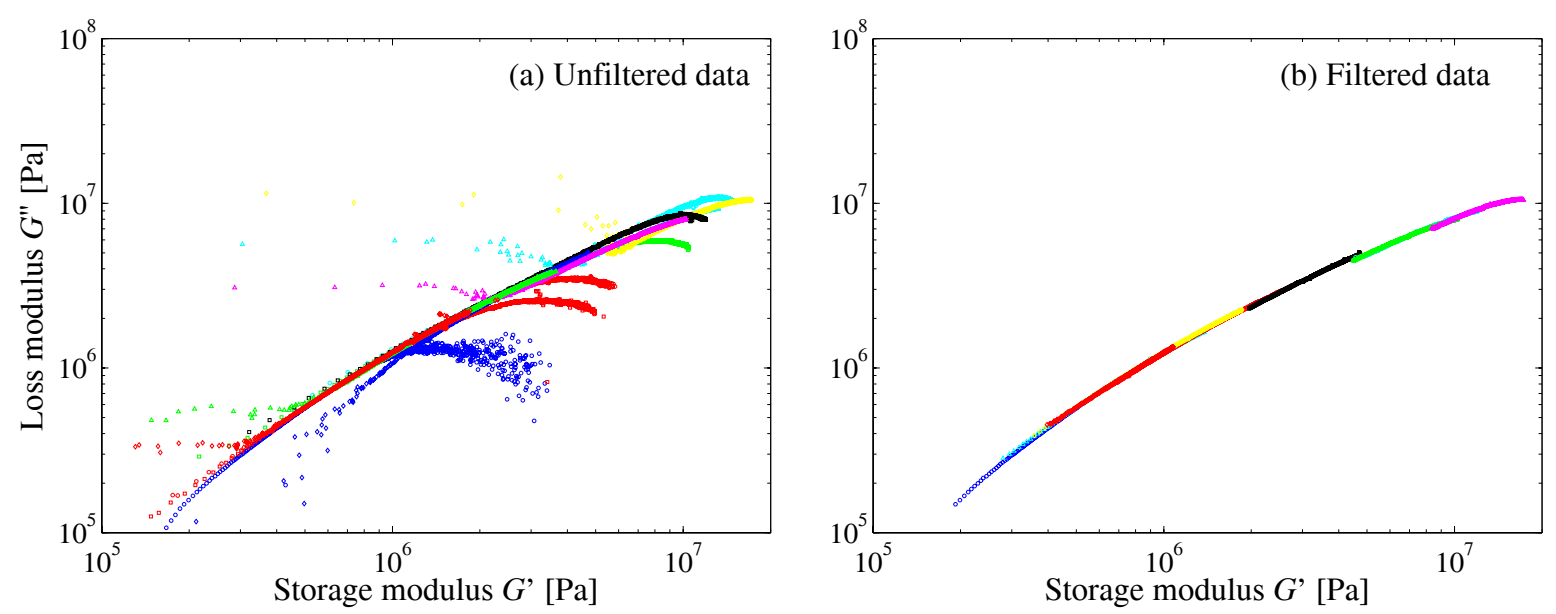

Figure 6: Wicket plot of the identified complex modulus functions (spectral filtering).

The set of complex modulus functions, identified at different temperatures, should finally be correlated to identify the shift factor $\alpha_{T}(T)$ distribution of the material by using the frequencytemperature equivalence principle. The shift factor relationship might be described by the Arrhenius equation as

$$
\log \left[\alpha_{T}(T)\right]=T_{A}\left(\frac{1}{T}-\frac{1}{T_{0}}\right),
$$

where $T$ is the absolute temperature of each data set, $T_{0}$ is the reference temperature and $T_{A}$ is identified by fitting the experimental data to the model. Using the identified shift factor distribution, the identified complex modulus functions can be represented in the unified and universal representation, the so-called reduced-frequency nomogram. This representation is very useful and broadly applied since it represents simultaneously the frequency and the temperature dependence of the complex modulus of a viscoelastic material by a single pair of curves: one for the storage modulus and another representing the loss factor distribution. Figure 7 overlaps the original nomogram of the 3M ISD112 viscoelastic material provided by the manufacturer [43] with the identified nomogram of the 3M ISD112 specimen under analysis. The identified nomogram agrees well with the one published by the viscoelastic material manufacturer and is consistent with other nomograms obtained with other specimens with different geometry using the same methodology. The reader is referred to the well-known textbooks of Nashif et al. and Jones $[1,4]$ for further details about the frequency-temperature equivalence principle and construction of the nomogram.

\section{PARAMETERS CURVE FITTING}

From the measured material properties (identified nomogram) of the 3M ISD112, and using the shift factor relationship previously determined, one can obtain the frequency dependent complex modulus for different temperature values. In order to use the internal variables viscoelastic damping models GHM and ADF defined in Sections 2.3.2 and 2.3.3, the characteristic model parameters (three and two for each GHM and ADF series, respectively) were determined using a curve-fitting technique based in the minimization (optimization procedure) of the least mean square error between the measured and predicted data, using the GHM and ADF definitions of the complex shear modulus in Equations (14) and (24), for a specified frequency range of interest. The number of series used in the GHM and ADF models determines how well the models are capable of matching the data over the frequency range of interest. 


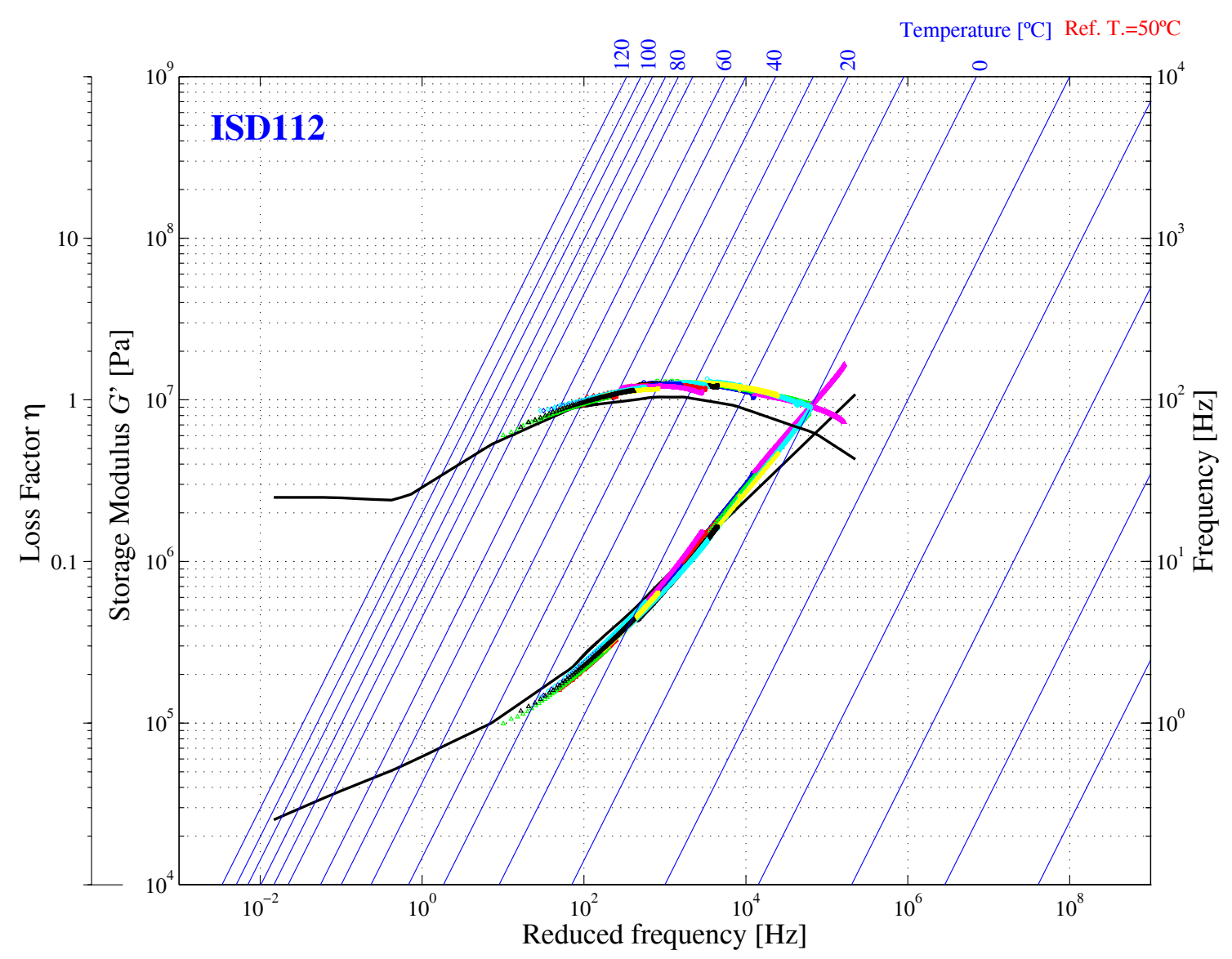

Figure 7: Nomogram of 3M ISD112: manufacturer's (black) and identified (color).

Table 2: Identified GHM and ADF parameters for material 3M ISD112 at $27^{\circ} \mathrm{C}$.

\begin{tabular}{|c|c|c|c|c|c|c|c|c|}
\hline \multicolumn{2}{|c|}{ Model } & \multicolumn{4}{|c|}{ GHM } & \multicolumn{3}{|c|}{ ADF } \\
\hline$n$ & $i$ & $G_{0}[\mathrm{MPa}]$ & $\hat{\alpha}_{i}$ & $\hat{\omega}_{i}$ & $\hat{\zeta}_{i}$ & $G_{0}[\mathrm{MPa}]$ & $\Delta_{i}$ & $\Omega_{i}$ \\
\hline 3 & 1 & 0.1633 & 4.8278 & 28045 & 22.013 & 0.1789 & 3.5286 & 504.20 \\
\hline & 2 & & 14.548 & 41494 & 3.1275 & & 8.7533 & 4286.5 \\
\hline & 3 & & 40.043 & 41601 & 0.6165 & & 60.324 & 39313 \\
\hline
\end{tabular}

For each viscoelastic model, one and three series of parameters were used to curve fit the identified complex modulus of the $3 \mathrm{M}$ ISD112 material at $27^{\circ} \mathrm{C}$ over the frequency range $[10-3000] \mathrm{Hz}$. The fitted curves of both models are presented in Figures 8 and 9 and compared with the measured data.

As can be verified, the quality of the fit depends on the number of terms (series) retained in the models. Using more series improves the accuracy of the models material description, however increasing the size of the problem. The values of the parameters determined in the fitting process for both GHM and ADF models are presented in Table 2. It's worthy to mention that the values of the parameters determined are guaranteed to define the material properties with accuracy only over the frequency range specified in the fitting process which in this case is $[10-3000] \mathrm{Hz}$. From the analysis of Figures 8 and 9 one can conclude that both models fit the measured data with a satisfactory accuracy with 3 series of parameters (one serie wouldn't be enough). 


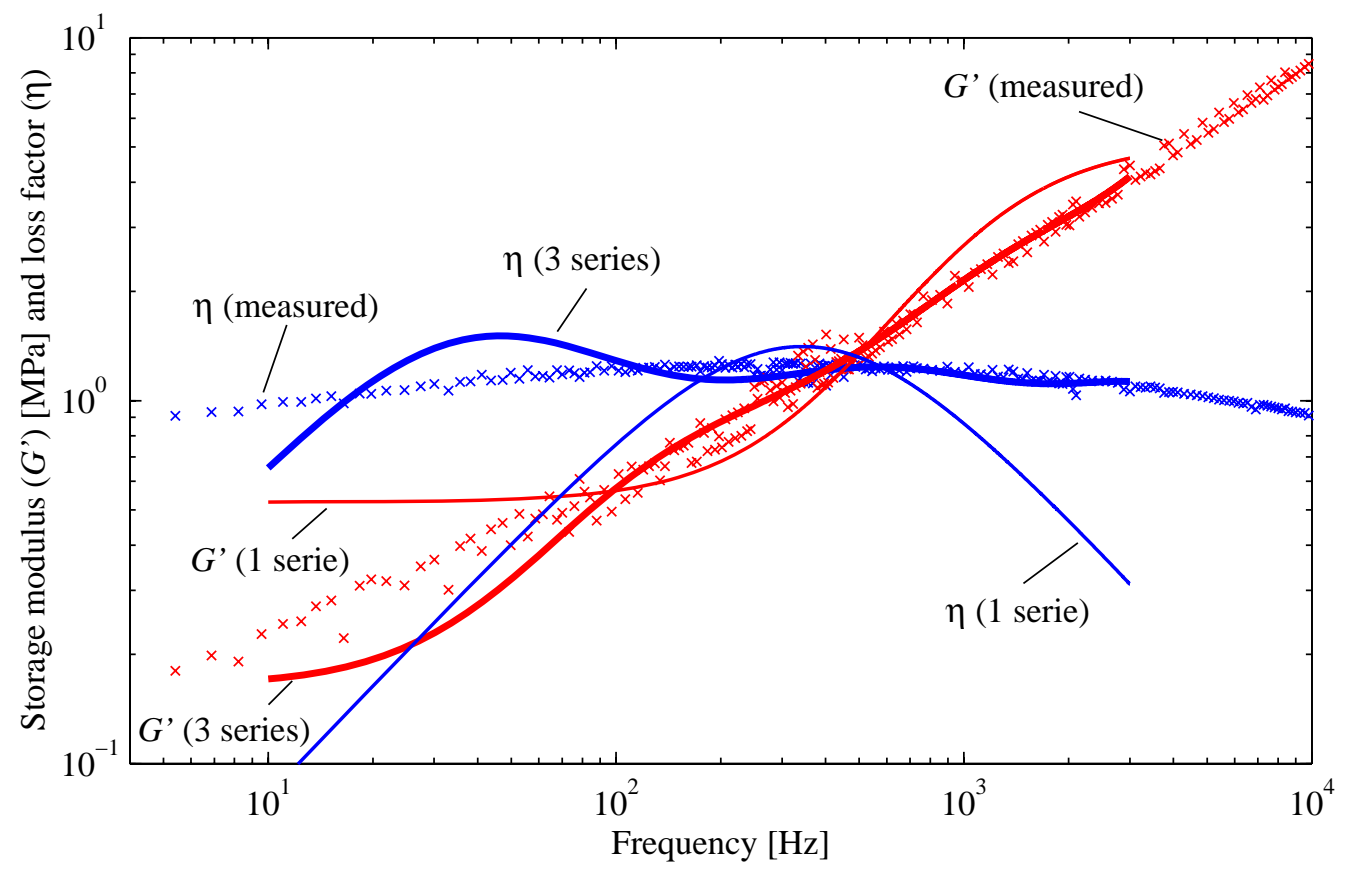

Figure 8: Curve fitted GHM curves with 1 and 3 series of parameters at $27^{\circ} \mathrm{C}$.

Table 3: Material properties of the viscoelastic sandwich plate specimen.

\begin{tabular}{lccc}
\hline \multicolumn{1}{c}{ Material } & $G[\mathrm{G} \mathrm{Pa}]$ & $\nu$ & $\rho\left[\mathrm{kg} \mathrm{m}^{-3}\right]$ \\
\hline AA 1050A H24 & 26.5 & 0.32 & 2708 \\
3M ISD112 & Figure 7 & 0.49 & 1140 \\
\hline
\end{tabular}

\section{VALIDATION OF THE DAMPING MODELS}

\subsection{Experimental specimen}

The aim of this section is to assess and validate the GHM and ADF viscoelastic damping models. Thus, a comparison of the FRFs of a viscoelastic sandwich plate predicted with the two GHM and ADF models and by a direct frequency analysis (DFA) approach (as described in section 2.3.1) and measured results is performed.

To develop the experimental study a sandwich plate specimen with an embedded viscoelastic damping treatment was produced. The viscoelastic material considered is the material $3 \mathrm{M}$ ISD112 [43] which was used to produce the viscoelastic layer applied in the core of the sandwich plate. The specimen was produced with aluminum plates (aluminum alloy 1050A H24) with each skin (plate) with dimensions $200 \times 100 \times 1 \mathrm{~mm}$. The properties of the viscoelastic material and aluminium applied in the specimen are presented in Table 3.

The viscoelastic sandwich specimen (Figure 10) was produced by sandwiching a 3M ISD112 viscoelastic layer with dimensions $200 \times 100 \times 0.127 \mathrm{~mm}$ between two identical aluminium plates. The specimen was produced following the manufacturer instructions and it was found that the 3M ISD112 can be easily bounded to the metallic substrate at room temperature [43].

\subsection{Experimental setup}

The purpose of this experimental study was to provide reference data for a comparison analysis with the adopted viscoelastic models results. Thus, the aim of the experimental study was the determination of a representative set of FRFs providing a reliable basis for comparison and 


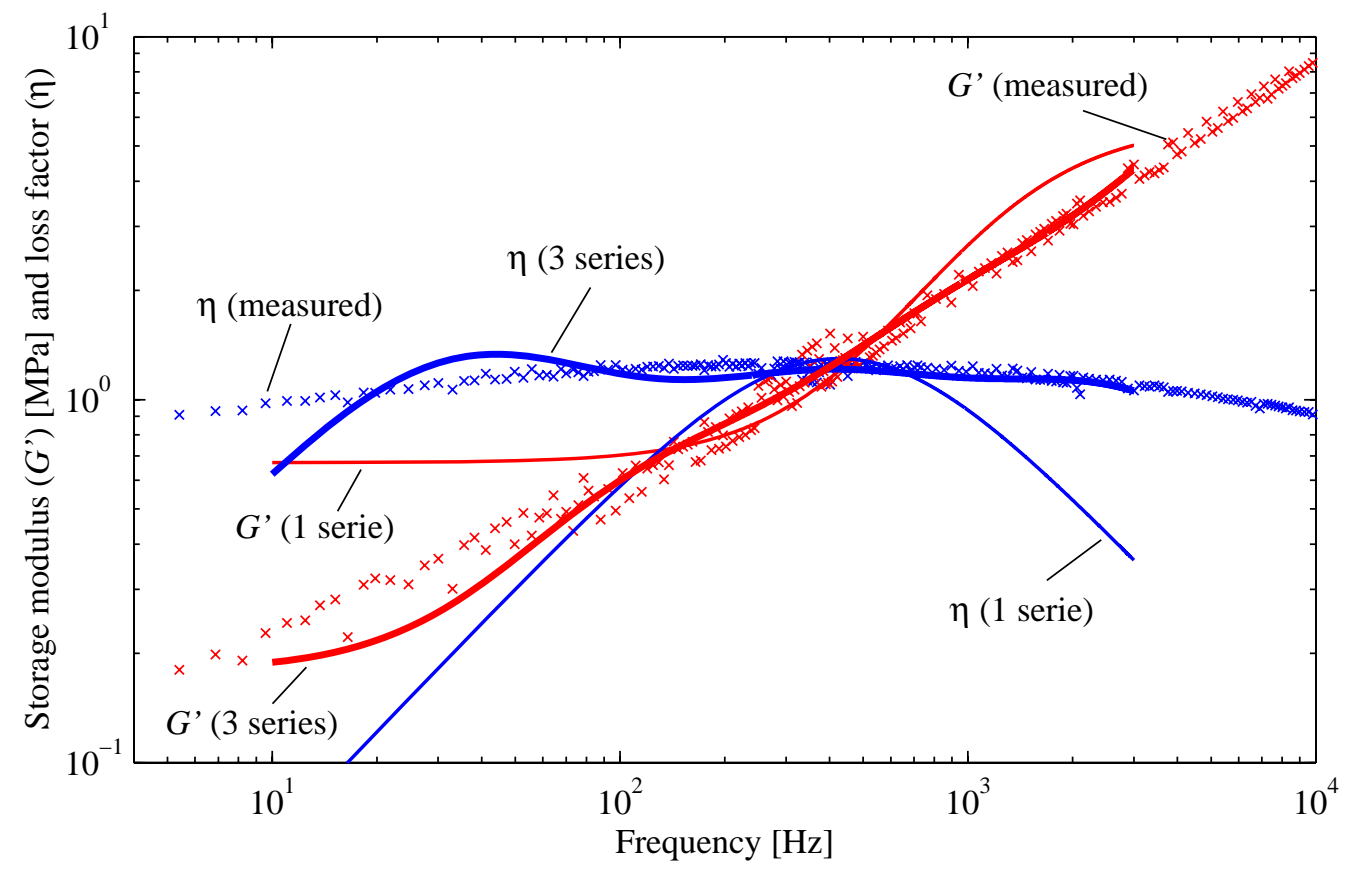

Figure 9: Curve fitted ADF curves with 1 and 3 series of parameters at $27^{\circ} \mathrm{C}$.

validation of the numerical models. The experimental specimen was suitably suspended in air by using a lightweight nylon wire from a rigid frame to approximate free boundary conditions, which minimize the boundary error effects. A grid with 15 points, as depicted in Figure 10, was defined for measurement. An electrodynamic shaker (Ling Dynamic Systems - model 201), suspended from an independent rigid frame, was utilized to generate a random $[0-800] \mathrm{Hz}$ excitation applied in point 5 of the specimen. A thin and flexible stinger was used to link the shaker to the miniature force transducer (Brüel \& Kjær - model 8203) attached to the plate surface, which provided the measurement of the applied dynamic force (Figure 11). The specimen response was evaluated by using a laser vibrometer (Polytec - model OFV303) to measure the velocity of each point of the measuring mesh (Figure 12). The FRFs determination was performed by a dynamic signal analyzer (Brüel \& Kjær - model 2035). The temperature was evaluated by a thermocouple located near the specimen. Fifteen FRFs (mobility functions) were determined for the specimen. However, here, for comparison of the damping models, only the driving point mobility FRF measured in point 5 is considered.

\subsection{Assessment and comparison of the results}

For comparison of results the driving point mobility FRF measured in point 5 of the mesh depicted in Figure 10 is utilized. The predicted FRFs were determined using a 4-node facet type quadrangular shell FE, based on a layerwise theory, developed for the dynamic modelling of laminated structures. The sandwich plate was modeled with a FE mesh discretization of $32 \times 16$ elements (the reader is referred to [53] for further details about the FE model utilized). The damping behavior of the viscoelastic sandwiched layer was incorporated by the three methods previously described: direct frequency analysis (DFA), where the stiffness matrix is re-calculated at each frequency value; the GHM and ADF models utilizing the three series of parameters identified (Table 2). The measured and predicted FRFs are compared in Figure 13. Results of the natural frequencies and damping ratios for the first 4 modes are presented in Table 4. 

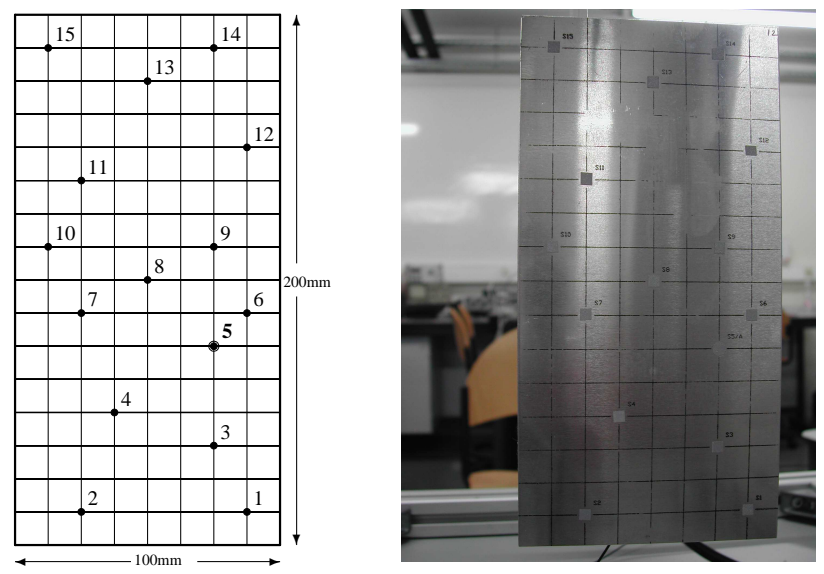

Figure 10: Measuring mesh and viscoelastic sandwich plate specimen.

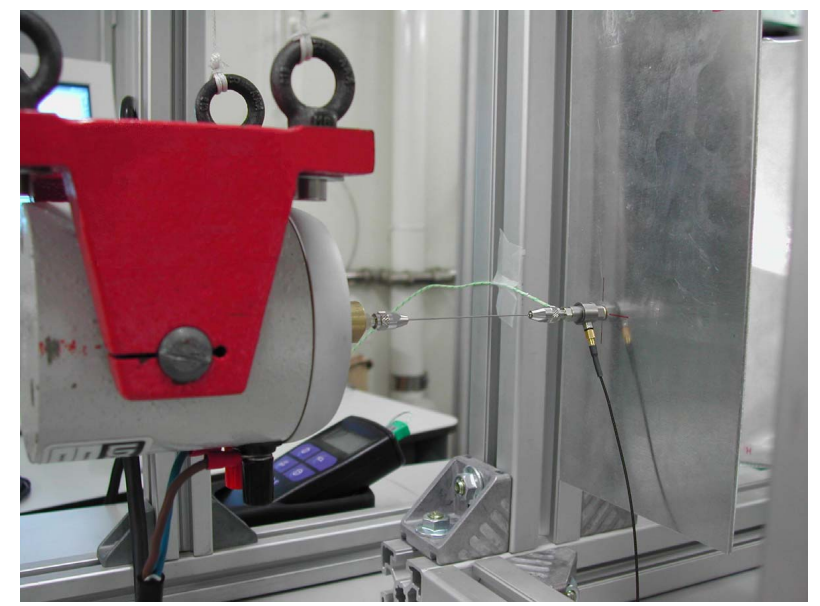

Figure 11: Experimental setup (specimen excitation).

From the results it can be observed that the predicted FRFs correlate well with the measured one. Both natural frequencies and damping ratios are well estimated with the two prediction methods, GHM and ADF models. It's worthy to mention that the DFA approach is very timeconsuming and doesn't provide directly the modal parameters of the viscoelastically damped structure. In opposition, the internal variables models provide an accurate mathematical model of the structure allowing, as in this specific case, to determine directly the natural frequencies and damping ratios from the extracted complex eigenvalues, identifying all the complex modes which sometime might be difficult to identify from the pos-processing of the FRFs. Last but not the least, the GHM and ADF models conduct to similar results, however, the size of the

Table 4: Measured and predicted natural frequencies [ $\mathrm{Hz}]$ and modal damping ratios [\%] of the first 4 modes of the viscoelastic sandwich plate.

\begin{tabular}{lcccc}
\hline & 1st mode & 2nd mode & 3rd mode & 4th mode \\
\hline Measured & $235.1(14.1)$ & - & $521.0(12.1)$ & - \\
Predicted (DFA) & $233.9(13.0)$ & - & $518.6(15.1)$ & - \\
Predicted (GHM) & $235.7(13.7)$ & $268.2(12.7)$ & $524.3(16.8)$ & $556.8(19.3)$ \\
Predicted (ADF) & $233.8(14.1)$ & $269.4(13.3)$ & $524.2(16.5)$ & $554.5(18.7)$ \\
\hline
\end{tabular}




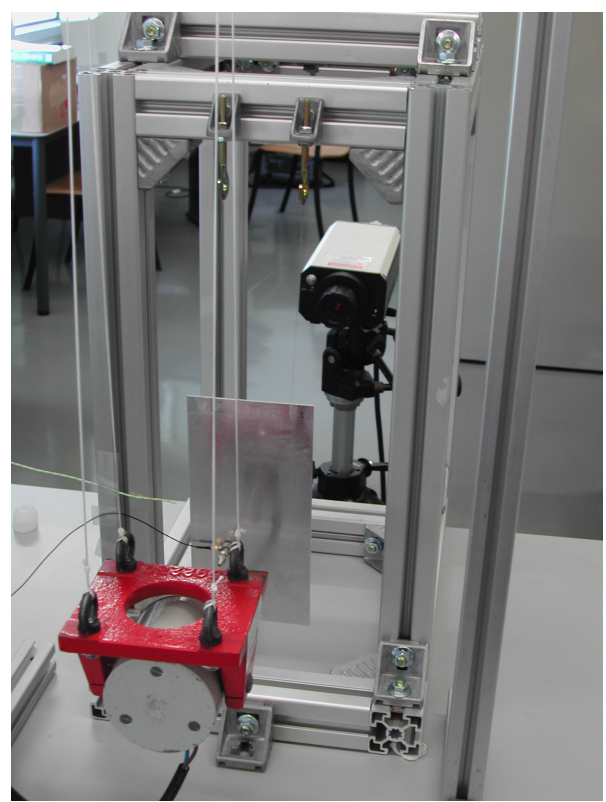

Figure 12: Experimental setup (response measurement).

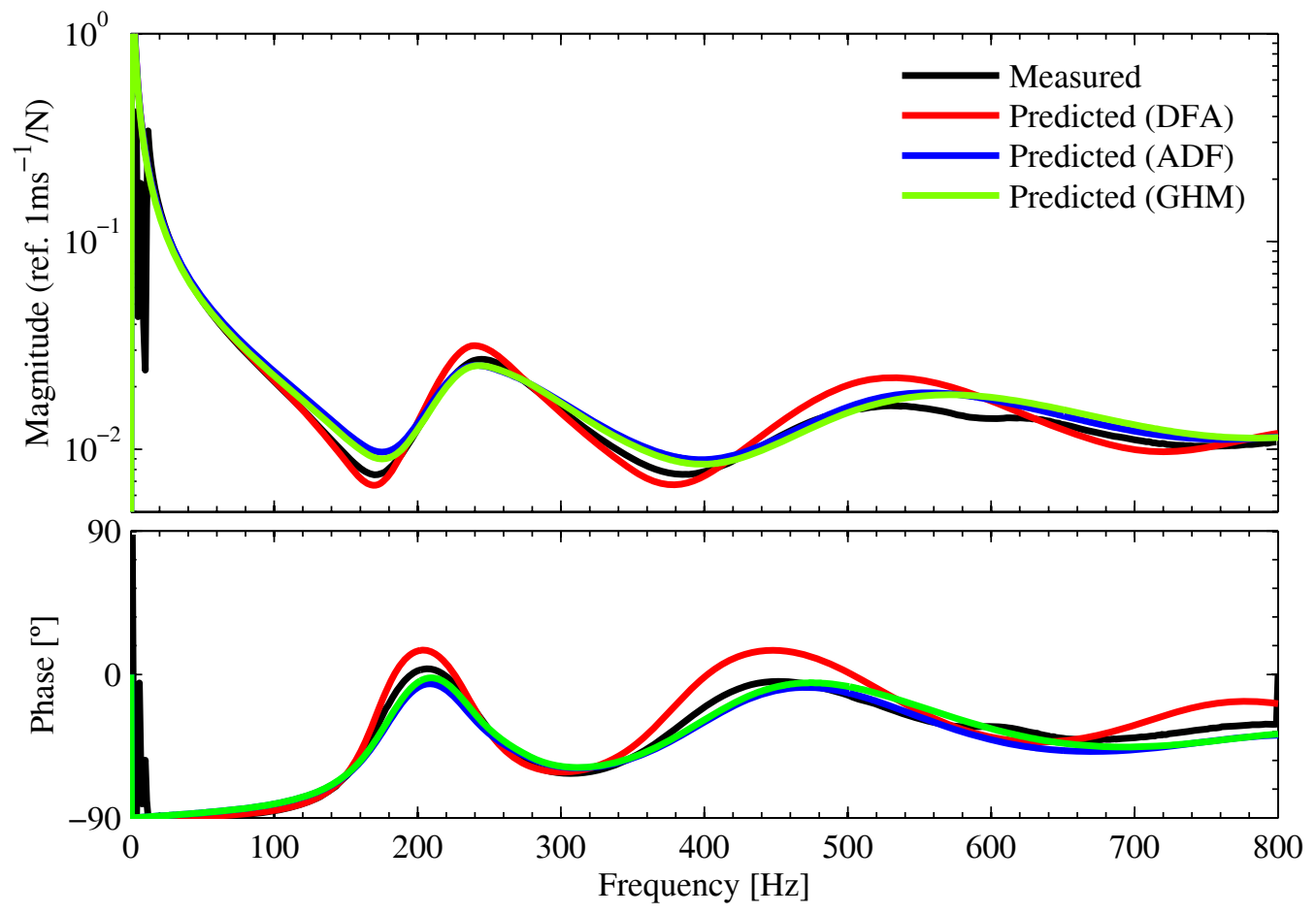

Figure 13: Direct mobility FRF of the viscoelastic sandwich plate at $27^{\circ} \mathrm{C}$ ).

problem with the ADF model is smaller and one concluded that the fitting process is easier to perform with the ADF model.

\section{CONCLUSION}

This paper has presented FE-based analytical strategies to model the damping behavior of viscoelastic materials which might be used (surface mounted, constrained or embedded) as 
damping treatments in structures. An experimental procedure to identify the complex shear modulus of viscoelastic materials was presented and the obtained data was used to fit the internal variables models GHM and ADF and identify their characteristic parameters for the 3M ISD112 viscoelastic material. Measured and FE-based predicted FRFs based on a direct frequency analysis (DFA), GHM and ADF models, were compared in order to assess the damping models and validate the experimental procedure for the material properties identification and the curve fitting process.

The application of a discrete dynamic system, describing a SDoF analytical model, provides a reliable identification methodology, since it is based on the direct (in opposition to indirect measuring approaches based on vibrating beams) characterization of the complex stiffness of a viscoelastic material sample in shear deformation, providing results similar to those published by the material manufacturer.

Regarding the internal variables models under analysis here, which were implemented at the FE model level, the ADF model is known to lead to an augmented model of the damped structural system with a lower size than the GHM model. To the opinion of the authors the ADF model represents the best alternative to accurately model the damping behavior since it yields good trade-off between accuracy and complexity. One major disadvantage in using internal variables models such as the GHM or ADF is the creation of additional dissipation (or anelastic) variables increasing the size of the coupled damped FE model. However, an alternative based on the direct frequency analysis (DFA) using the FE spatial model and re-calculating the complex viscoelastic stiffness matrix for each discrete frequency value might be used with the outcome of being simpler to implement and the drawback of being time-consuming and not providing directly the modal parameters of the damped structural system. All the models have similar accuracy and yield representative results of viscoelastically damped structural systems.

\section{ACKNOWLEDGMENTS}

The funding given by Fundação para a Ciência e a Tecnologia of the Ministério da Ciência e da Tecnologia of Portugal under grant POSI SFRH/BD/13255/2003 is gratefully acknowledged by the first author. Acknowledgements are also addressed to 3M Company for providing the viscoelastic materials used in this work.

\section{REFERENCES}

[1] A. Nashif, D. Jones and J. Henderson. Vibration Damping. John Wiley \& Sons, New York, NY, 1985.

[2] C. T. Sun and Y. P. Lu. Vibration Damping of Structural Elements. Prentice Hall, Englewood Cliffs, NJ, 1995.

[3] D. J. Mead. Passive Vibration Control. John Wiley \& Sons, Chichester, UK, 1998.

[4] D. I. G. Jones. Handbook of Viscoelastic Vibration Damping. John Wiley \& Sons, Chichester, UK, 2001.

[5] C. H. Park and A. Baz. Vibration damping and control using active constrained layer damping: A survey. The Shock and Vibration Digest, 31(5):355-364, 1999.

[6] A. Benjeddou. Advances in hybrid active-passive vibration and noise control via piezoelectric and viscoelastic constrained layer treatments. Journal of Vibration and Control, 7 (4):565-602, 2001. 
[7] M. A. Trindade and A. Benjeddou. Hybrid active-passive damping treatments using viscoelastic and piezoelectric materials: Review and assessment. Journal of Vibration and Control, 8(6):699-745, 2002.

[8] R. Stanway, J. A. Rongong and N. D. Sims. Active constrained-layer damping: A stateof-the-art review. Proceedings of the Institution of Mechanical Engineers. Part I: Journal of Systems and Control Engineering, 217(6):437-456, 2003.

[9] B. Azvine, G. R. Tomlinson and R. J. Wynne. Use of active constrained-layer damping for controlling resonant vibration. Smart Materials and Structures, 4(1):1-6, 1995.

[10] J. Ro, A. El-Ali and A. Baz. Control of sound radiation from a fluid-loaded plate using active constrained layer damping. In N. S. Ferguson, H. F. Wolfe and C. Mei, editors, Proceedings of the Sixth International Conference on Recent Advances in Structural Dynamics, pages 1252-1273, Southampton, UK, 1997.

[11] M. C. Ray and A. Baz. Optimization of energy dissipation of active constrained layer damping treatments of plates. Journal of Sound and Vibration, 208(3):391-406, 1997.

[12] C. H. Park and A. Baz. Vibration control of bending modes of plates using active constrained layer damping. Journal of Sound and Vibration, 227(4):711-734, 1999.

[13] Q. Liu, A. Chattopadhyay, H. Gu and X. Zhou. Use of segmented constrained layer damping treatment for improved helicopter aeromechanical stability. Smart Materials and Structures, 9(4):523, 2000.

[14] A. Baz. Active constrained layer damping of thin cylindrical shells. Journal of Sound and Vibration, 240(5):921-935, 2001.

[15] C. Chantalakhana and R. Stanway. Active constrained layer damping of clamped-clamped plate vibrations. Journal of Sound and Vibration, 241(5):755, 2001.

[16] S. H. Ko, C. H. Park, H. C. Park and W. Hwang. Vibration control of an arc type shell using active constrained layer damping. Smart Materials and Structures, 13(2):350, 2004.

[17] C. M. A. Vasques, B. R. Mace, P. Gardonio and J. D. Rodrigues. Arbitrary active constrained layer damping treatments on beams: Finite element modelling and experimental validation. Computers and Structures, 2005 (in press).

[18] C. M. A. Vasques and J. Dias Rodrigues. Adaptive feedforward control of vibration of a beam with active-passive damping treatments: Numerical analysis and experimental implementation. In Proceedings of the 16th International Conference on Adaptive Structures and Technologies (ICAST 2005), pages 255-262. DEStech Publications, Paris, France, 2006.

[19] R. A. Moreira and J. D. Rodrigues. The modelisation of constrained damping layer treatment using the finite element method: Spatial and viscoelastic behavior. In Proceedings of the International Conference on Structural Dynamics Modelling: Test, Analysis Correlation and Validation, Madeira, Portugal, 2002.

[20] R. Moreira and J.D. Rodrigues. Constrained damping layer treatments: Finite element modeling. Journal of Vibration and Control, 10(4):575-595, 2004.

[21] C. D. Johnson, D. A. Kienholz and L. C. Rogers. Finite element prediction of damping in beams with constrained viscoelastic layers. Shock and Vibration Bulletin, (1):71-81, 1980. 
[22] M. A. Trindade, A. Benjeddou and R. Ohayon. Modeling of frequency-dependent viscoelastic materials for active-passive vibration damping. Journal of Vibration and Acoustics, 122(2):169-174, 2000.

[23] A. R. Johnson. Modeling viscoelastic materials using internal variables. Shock and Vibration Digest, 31(2):91-100, 1999.

[24] D. F. Golla and P. C. Hughes. Dynamics of viscoelastic structures a time-domain, finite element formulation. Jounal of Applied Mechanics, 52(12):897-906, 1985.

[25] D. J. McTavish and P. C. Hughes. Modeling of linear viscoelastic space structures. Journal of Vibration and Acoustics, 115(1):103-110, 1993.

[26] G. A. Lesieutre and E. Bianchini. Time domain modeling of linear viscoelasticity using anelastic displacement fields. Journal of Vibration and Acoustics, 117(4):424-430, 1995.

[27] G. A. Lesieutre, E. Bianchini and A. Maiani. Finite element modeling of one-dimensional viscoelastic structures using anelastic displacement fields. Journal of Guidance Control and Dynamics, 19(3):520-527, 1996.

[28] Y.C. Yiu. Finite element analysis of structures with classical viscoelastic materials. In 34th AIAA/ASME/ASCE/AHS/ASC Structures, Structural Dynamics and Materials Conference, volume 4, pages 2110-2119, La Jolla, CA, USA, 1993.

[29] L. A. Silva. Internal Variable and Temperature Modeling Behavior of Viscoelastic Structures - A Control Analysis. PhD thesis, Virginia Tech, VA, USA, 2003.

[30] R. L. Bagley and P. J. Torvik. Fractional calculus - A different approach to the analysis of viscoelastically damped structures. AIAA Journal, 21(5):741-748, 1983.

[31] R. L. Bagley and P. J. Torvik. Fractional calculus in the transient analysis of viscoelastically damped structures. AIAA Journal, 23(6):918-925, 1985.

[32] S. Adhikari and J. Woodhouse. Quantification of non-viscous damping in discrete linear systems. Journal of Sound and Vibration, 260(3):499-518, 2003.

[33] C. R. Brackbill, G. A. Lesieutre, E. C. Smith and K. Govindswamy. Thermomechanical modeling of elastomeric materials. Smart Materials \& Structures, 5(5):529-539, 1996.

[34] G. A. Lesieutre and K. Govindswamy. Finite element modeling of frequency dependent and temperature-dependent dynamic behavior of viscoelastic materials in simple shear. International Journal of Solids and Structures, 33(3):419-432, 1996.

[35] A. Baz. Robust control of active constrained layer damping. Journal of Sound and Vibration, 211(3):467-480, 1998.

[36] M. I. Friswell and D. J. Inman. Hybrid damping treatments in thermal environments. In G.R. Tomlinson and W.A. Bullough, editors, Smart Materials and Structures, pages 667-674. IOP Publishing, Bristol, UK, 1998.

[37] M. A. Trindade, A. Benjeddou and R. Ohayon. Finite element analysis of frequency- and temperature-dependent hybrid active-passive vibration damping. Revue Européenne des Éléments Finis, 9(1-3):89-111, 2000.

[38] L. A. Silva, E. M. Austin and D. J. Inman. Time-varying controller for temperaturedependent viscoelasticity. Journal of Vibration and Acoustics, 127(3):215-222, 2005. 
[39] V. Pradeep and N. Ganesan. Vibration behavior of acld treated beams under thermal environment. Journal of Sound and Vibration, 292(3-5):1036-1045, 2006.

[40] M. L. Drake and J. Soovere. A design guide for damping of aerospace structures. In AFWAL Vibration Damping Workshop, volume 3, 1984.

[41] M. L. Drake and G. E. Terborg. Polymeric material testing procedures to determine damping properties and the results of selected commercial materials. Technical Report AFWALTR-80-4093, Materials Laboratory, Wright-Patterson Air Force Base, 1980.

[42] M. L. Drake. Damping properties of various materials. Technical Report AFWAL-TR-884248, Materials Laboratory, Wright-Patterson Air Force Base, 1988.

[43] 3M. Scotchdamp Vibration Control Systems: Product Information and Performance Data. 3M Industrial Tape and Specialties Division, St. Paul, MN, USA, 1993.

[44] M. I. Friswell, D. J. Inman and M. J. Lam. On the realisation of GHM models in viscoelasticity. Journal of Intelligent Material Systems and Structures, 8(11):986-993, 1997.

[45] M. Enelund and G. A. Lesieutre. Time domain modeling of damping using anelastic displacement fields and fractional calculus. International Journal of Solids and Structures, 36(29):4447-4472, 1999.

[46] R. M. Christensen. Theory of Viscoelasticity: An Introduction. Academic Press, New York, 2nd edition, 1982.

[47] C. H. Park, D. J. Inman and M. J. Lam. Model reduction of viscoelastic finite element models. Journal of Sound and Vibration, 219(4):619-637, 1999.

[48] G. A. Lesieutre. Finite elements for dynamic modeling of uniaxial rods with frequencydependent material properties. International Journal of Solids and Structures, 29(12): 1567-1579, 1992.

[49] C. M. A. Vasques, B. Mace, P. Gardonio and J. D. Rodrigues. Analytical formulation and finite element modelling of beams with arbitrary active constrained layer damping treatments. Institute of Sound and Vibration Research, Technical Memorandum TM934, 2004.

[50] M. I. Friswell and D. J. Inman. Reduced-order models of structures with viscoelastic components. AIAA Journal, 37(10):1318-1325, 1999.

[51] C. M. A. Vasques and J. Dias Rodrigues. Simulation of combined feedback/feedforward active control of vibration of beams with ACLD treatments. Computers and Structures, 2006 (accepted).

[52] B. R. Allen. A direct complex stiffness test system for viscoelastic material properties. In Proceedings of 3rd Smart Structures and Materials (SPIE), volume 2720, pages 338-345, San Diego, CA, USA, 1996.

[53] R. A. S. Moreira, J. D. Rodrigues and A. J. M. Ferreira. A generalized layerwise finite element for multi-layer damping treatments. Computational Mechanics, 37(5):426-444, 2006. 